\title{
Analysis of MPSO-Successive Interference Cancellation Multipath Routing Protocol
}

\author{
Santosh Verma* \\ M.Tech Scholar Department of CSE TIT, Bhopal, India.
}

*Corresponding Author: Santosh Verma, M.Tech Scholar Department of CSE TIT, Bhopal, India.

\begin{abstract}
Wireless Sensor Networks has a greater advantage in today 's communication application such as environmental, traffic, military, health monitoring. While considering adhoc network in sensitive areas, one of the important aspect to consider is energy because while transmitting data all communicating nodes exhausts its battery life. For mobile nodes in adhoc scenario one and only one source of energy is battery. As compared to single path multipath routing helps in finding optimal path that requires less energy and enhances the network lifetime. In this research an energy efficient routing protocol is proposed. In routing algorithm, route that have shortest path among multipaths selected by modified particle swarm optimized Successive Interference Cancellation algorithm.
\end{abstract}

Keywords: Adhoc network, Routing protocols, Throughput, Bandwidth Aware, SIC, MPSO.

\section{INTRODUCTION}

Recent developments in ad hoc wireless technologies have allowed wireless sensor networks to create spontaneous connections between devices with or without infrastructures [1,2]. In addition, with the emergence of intelligent sensor-driven mobile devices, WSNs have become an essential part of the infrastructure of smart cities and the Internet of Things, with intelligent devices that can be customized and automatically configured by the WSN. send, receive and share data in an extremely restricted area (see Figure 1.2) [3].

In a highly intelligent environment, Wireless Sensor Networks (WSN) and Wireless Mesh Networks (WMN) are key technologies that provide many IoT applications and services to users. In addition, WSN has found a wide range of applications in the fields of health, battlefield communications, disaster recovery, crisis management organizations, ad hoc cooperative IT, social activities and conference rooms.

Despite the interesting applications of WSN, these systems still have to face numerous challenges and constraints that require further investigation before the commercial diffusion of WSN. The most important constraints on the design of WSN are: 1) limited battery life and duration, quality of service (QoS), configuration without infrastructure and dynamic network topologies, node mobility, reliability of wireless connections, functionality of variable nodes, scalability multi-hop routing, multicast support and security threats [4].

Therefore, the routing protocol plays an important role in these networks and the WSN constraints remain in the development of the latest routing protocols to modify the efficient transmission of packets on wireless support, mainly once the source and the destination are non-adjacent nodes. The routing protocol should choose the best path between source-destination node pairs in terms of power consumption and quality of service measurements such as available bandwidth, average end-to-end delay, packet loss and noise average. The reminder of this document is as follows.

Various approaches to high-speed, ad-hoc, and bandwidth multi-hop routing algorithms have been studied and adopted to reduce latency, delay and bandwidth of the connection and ensure a guaranteed level of application performance. sensitive to the quality of the service. Multipath routing is more promising in ad hoc networks because it provides additional features such as load balancing, fault tolerance, higher throughput, etc. To ensure quality of service in ad networks. hoc. Most of the routing protocols proposed for mesh and ad hoc networks are unipath, which means that only one route is used between a source node and a destination node. The main goal of multipath routing is to allow the use 
of numerous valid paths to reach destinations, not just the best way. This should be done without imposing excessive control over the maintenance of these routes.

The availability of multiple paths between an origin and a destination can be used to obtain the following benefits:

Fault Tolerance: the introduction of redundancy in the network or the provision of backup paths to be used in the event of a fault [4] are forms of introducing fault tolerance at routing level in mesh networks. To this end, some techniques can be applied as packet recovery [5], which involves modifying the path of a packet if the actual path is interrupted.

Improved throughput: In a mesh network, some connections may have limited bandwidth. Single-route routing may not provide enough bandwidth for a connection. Therefore, using multiple paths simultaneously to route data can be a good approach to meeting the bandwidth requirements of some applications. By increasing throughput, you get a reduced end-to-end delay and improve service quality [6].

\section{RELATED WORK}

In [2], Perkins et al. used a flooding technique to find a shortest route but it is not always useful for energy consumption where detected routes have no sufficient energy to send data between a source and the destination. Otherwise, we always assume that the energy capacity of nodes is a critical resource and it should be consumed in a way to ensure a longest lifetime of the network.

In [2], AlShawi et al. proposed a new routing method for WSNs to extend network lifetime using a combination of a fuzzy approach and an A-star algorithm. The proposed method aims to determine an optimal routing path from the source to the destination by favoring the highest remaining battery power, minimum number of hops and lowest traffic load, and balancing between them in order to extend the network lifetime as much as possible. However, this method requires a localization support which imposes significant overhead in terms of communication and computational complexity.

The approaches in [4], Montoya et al. implemented a multi-path technique to balance the power consumption in order to extend the lifetime of the WSN. The proposed heuristic technique implements a mechanism to construct a path, choosing the next communicating sensor node according to its distance and Received Signal Strength Indicator (RSSI) level. Additionally, while a path is constructed, the algorithm avoids cycles and has the ability to reconstruct the path once the discovering route has arrived to a leaf. As soon as several paths from the same target reach a particular sink, this node performs an energy balancing strategy which consists in deciding on the amount of flow to transmit on each of the paths.

In [5], Kacimi presented two approaches. First, a traffic load balancing technique is used to optimize the energy consumption of the nodes in a grid topology with a base station at a corner. Subsequently, a distributed heuristic algorithm is proposed to combine load balancing with transmission power control in order to find the good traffic proportions between the nodes to ensure a best balance of their energy consumption. However, this method works only for a grid topology.

In [6], Jha et al. dicussed about the working of proposed energy efficient bandwidth aware shortest path routing protocol for multipath routing in wireless sensor network. The proposed algorithm is based for choosing energy efficient shortest path. In routing algorithm, route that have shortest path among multipaths selected by particle swarm optimization algorithm. Among these shortest paths, that path is selected which require minimum route selection parameter.

In [7] Ming et al. proposed an Energy-Efficient Multi-path Routing Protocol (EEMRP), which searches multiple node-disjoint paths and employs a load balancing method to assign the traffic over each selected path. Both the residual energy level of nodes and the number of hops are considered to be incorporated into the link cost function. The link cost function is used by the node to select the next hop during the path search phase. Furthermore, since EEMRP only takes care of data transfer delays, the reliability of successful paths is sometimes limited.

In [8], Liu et al. propose an Energy Balancing and unequal Clustering Algorithm (EBCAG), which partitions the nodes into clusters of unequal size, where each sensor node maintains a gradient value, which is defined as its minimum hop count to the sink. The size of a cluster is decided by the gradient value of its cluster head, and the data gathered from the cluster members should follow the direction of the descending gradient to reach the sink. However, this approach is based on a WSN with uniform 
distribution. In some real-world applications, the approach of uniform sensor distribution may not be technically or practically feasible.

In [9], Abdelkader et al proposed a multipath routing algorithm based on the residual energy of the network. This setting is based on the number of hops in each node to find the easiest path and place it in the routing table. The main idea of this algorithm comes from the ant colony optimization (ACO).

\section{MeTHOdOLOGY}

What we describe here is our contribution to define multiple routes between a given node $n_{i}$ and the source node (eventually the sink node) by selecting a subset of all existing routes. The defined technique is inspired from two concepts one is particle swarm optimization as well as successive interference cancellation.

The flow chart of proposed algorithm is discussed below:

Steps of Proposed Algorithm

Step 1: Send RREQ from source node

Step 2: Receive route reply from neighbor nodes

Step 3: Establish different paths from source to destination

Step 4: Calculate ' $n$ ' shortest paths using successive interference cancellation

Step 5: Choose one among all shortest paths using modified PSO algorithm

Step 6: Reserve remaining shortest path for future reference

Step7: Forward Data packets

Successive interference cancellation (SIC)

Successive interference cancellation (SIC) may be a well-known physical layer technique [13]. Briefly, sic is that the ability of a receiver to receive 2 or more signals at the same time (that otherwise cause a collision in today's systems). attack is feasible as a result of the receiver could also be able to decode the stronger signal, deduct it from the combined signal, and extract the weaker one from the residue.

Our key observations could also be summarized as follows:

In the case of distinct receivers $(\mathrm{T} 1 \rightarrow \mathrm{R} 1$ and $\mathrm{T} 2 \rightarrow \mathrm{R} 2)$, the gains from sic are marginal.

In the case of common receivers $(\mathrm{T} 1 \rightarrow \mathrm{R} 1 \leftarrow \mathrm{T} 2)$, sic could provide modest MAC layer throughput gains if transmitters are rigorously coordinated with techniques like transmitter pairing and power reduction. However, somewhat counter-intuitively, the throughput gain is maximized once the system is forced to operate below the physical (PHY) layer capability.

We notice that these behaviors hold even below numerous real-world network architectures (e.g., enterprise WLANs, wherever multiple APs are connected via a wired backbone).

$$
S I C=\frac{P(t x) d^{-\alpha}(i, j)}{\sum_{k=1}^{N} P(t x) d^{-\alpha}(i, j)+\sigma^{2}}
$$

Where, $P(t x)=$ Transmission Power

$\mathrm{d}^{-\alpha}(\mathrm{i}, \mathrm{j})=$ Distance between node $\mathrm{i}$ and $\mathrm{j}$

$\sigma=$ Power level of noise

$\alpha=$ Path loss components

Successive interference cancellation (SIC) is a physical layer capability that allows a receiver to decode packets that arrive simultaneously. While the technique is well known in communications literature, emerging software radios are making practical experimentation feasible [14]. This motivates us to study the extent of throughput gains possible with SIC.

\section{Modified PSO}

- Begin, initialize the parameters, No of particles (Np), Maximum Iteration (Itrmax), Cognitive factor (C1), Social Factor (C2), Maximum Particle Velocity (velmax), Minimum Particle Velocity (velmin)

- Initialize Np

- Calculate fitness function of all particles, $\operatorname{Ff}(\mathrm{Np})$

- Initialize Pbest and Gbest

International Journal of Research Studies in Computer Science and Engineering (IJRSCSE) 
- $\quad$ for $\mathrm{t}=1$ : $\operatorname{Tmax}$

- Calculate the inertia weight Iw as

- $W=W_{\max }-\left(W_{\max }-W_{\min }\right)\left(\frac{t}{I t r_{\max }}\right)$

- Wmax and Wmin are the maximum and minimum inertia weight

- $\mathrm{t}=$ number of iteration

- for $\mathrm{i}=1: \mathrm{Np}$

- for $\mathrm{d}=1: \mathrm{D}$

- Update the velocity of particles $(\mathrm{Vt}+1)$

- Velnew $=$ Velold $+\mathrm{c} 1 * \mathrm{r} 1 *($ Pbest - Present_Position $)+\mathrm{C} 2 * \mathrm{R} 2 *$ (Gbest - Present_Position $)$

- Present_Position $=$ Present Position + Velold

- Convert the velocity into probability value

- $S\left(V e l_{t+1}\right)=\frac{1}{1+\exp \left(-V e l_{t+1}\right)}$

- Update the position of particle, $\mathrm{X} \mathrm{t+1}$ id using Equation (10)

- Present $_{\text {Position }_{t+1}}=1$, if $S\left(\right.$ Vel $\left._{t+1}\right)>$ rand

- Present $_{\text {Position }_{t+1}}=0$, otherwise

- increment d

- Calculate fitness function of new particles, $\mathrm{Ff}(\mathrm{Nt}+1)$

- increment i

The Advantages of Proposed Approach are End to End Delay is less, Energy consumption is reduced due to fact that the routes that are discovered are very less.

\section{Result Analysis}

In this research work the MATLAB tool is used to simulate and verify the validity of MPSO-SIC optimized multipath routing protocol. The result analysis is performed with variable number of packets. The residual energy is calculated as:

Depending on the distance between transmitter and receiver, multiple fade and free space channel patterns are used. The free space model (loss of power, $\mathrm{d}^{2}$ ) is mainly used for communication, while the power loss model $\mathrm{d}^{4}$ is used for communication between nodes. The radio energy consumed by the transmitter to transmit a 1 bit message at a distance $d$ is:

$$
\begin{aligned}
\mathrm{E}_{\mathrm{Tx}}(\mathrm{I}, \mathrm{d}) & =\mathrm{E}_{\mathrm{Tx}-\text { elec }}(\mathrm{I})+\mathrm{E}_{\mathrm{TX}-\mathrm{amp}}(\mathrm{I}, \mathrm{d}) \\
& =\mathrm{IE}_{\text {elec }}+\mathrm{IE}_{\mathrm{amp}} \mathrm{d}^{4}
\end{aligned}
$$

And energy consumed by the receiver is

$$
\mathrm{E}_{\mathrm{Rx}}(\mathrm{I})=\mathrm{E}_{\mathrm{Rx}-\mathrm{elec}}(\mathrm{I})=\mathrm{IE}_{\text {elec }}
$$

Where,

$E_{\text {elec }}=$ Per bit energy consumed to execute transmitter and receiver $E_{a m p}=$ amplifier energies

The simulation parameters are given below in table I.

Table1. Simulation Parameters

\begin{tabular}{|l|l|}
\hline Parameter Name & Values \\
\hline Network Area & $100 * 100$ \\
\hline Number of nodes & 100 \\
\hline Packet Size & $4000 \mathrm{bits}$ \\
\hline No. of packets & $1-500$ \\
\hline Initial Energy, Eo & $.5 \mathrm{~J}$ \\
\hline Transmitter Energy, ETX & $50 \mathrm{~nJ} / \mathrm{bit}$ \\
\hline Receiver Energy, ERX & $50 \mathrm{~nJ} / \mathrm{bit}$ \\
\hline Amplification Energy for short distance, Efs & $10 \mathrm{pJ} / \mathrm{bit} / \mathrm{m}^{2}$ \\
\hline Amplification Energy for long distance, Emp & $0.0013 \mathrm{pJ} / \mathrm{bit} / \mathrm{m}^{2}$ \\
\hline
\end{tabular}

According to proposed algorithm following results are analyzed as following: 


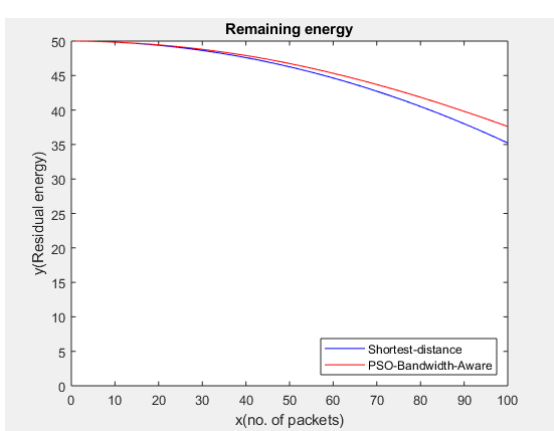

Figure1. Residual Energy Analysis with 100 Packets

In figure 1, the result analysis is performed for 100 packets and it is concluded that MPSO-SIC optimized multipath routing protocol outperforms better with respect to shortest multipath routing protocol.

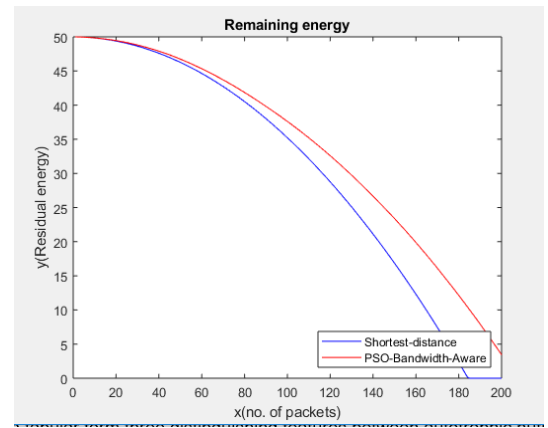

Figure2. Residual Energy Analysis with 200 Packets

In figure 2, the result analysis is performed for 200 packets and it is concluded that MPSO-SIC optimized multipath routing protocol outperforms better with respect to shortest multipath routing protocol.

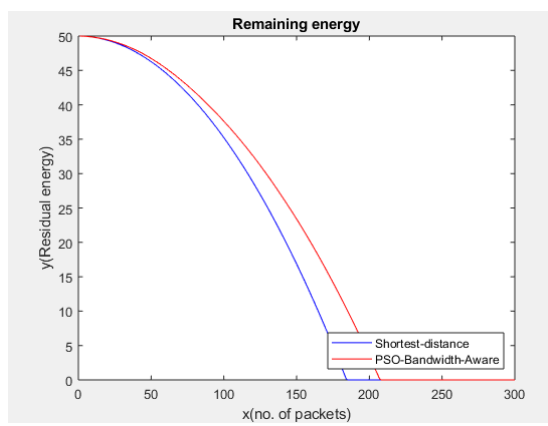

Figure3. Residual Energy Analysis with 300 Packets

In figure 3, the result analysis is performed for 300 packets and it is concluded that MPSO-SIC optimized multipath routing protocol outperforms better with respect to shortest multipath routing protocol.

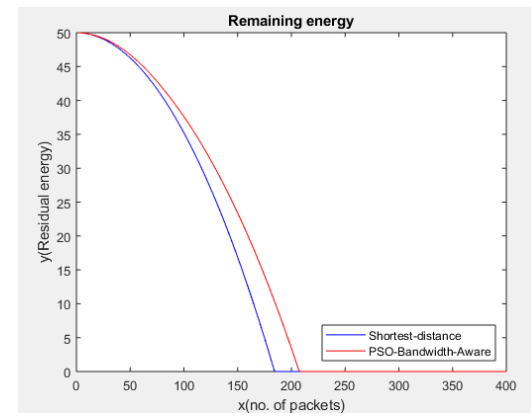

Figure4. Residual Energy Analysis with 400 Packets

In figure 4, the result analysis is performed for 400 packets and it is concluded that MPSO-SIC optimized multipath routing protocol outperforms better with respect to shortest multipath routing protocol. 


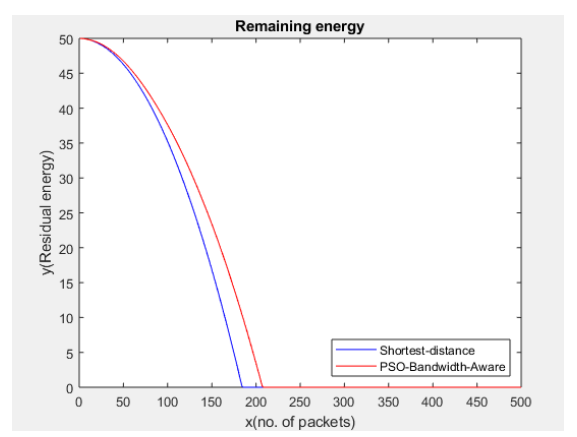

Figure5. Residual Energy Analysis with 500 Packets

In figure 5, the result analysis is performed for 500 packets and it is concluded that MPSO-SIC optimized multipath routing protocol outperforms better with respect to shortest multipath routing protocol.

\section{CONCLUSION}

The proposed algorithm uses SIC value of nodes as a parameter to find optimum paths using modified particle swarm optimization. Among these selected paths, only one optimum path is selected which reduces the energy requirement of the network. The MPSO-SIC algorithm selects energy efficient route among different shortest paths. The performance of the proposed algorithm is compared with multipath AODV routing protocol and concluded that PSO optimized energy efficient path is more efficient with respect to remaining energy of the network.

\section{REFERENCES}

[1] Bansal, M., Rajput, R., \& Gupta, G., "Mobile ad hoc networking (MANET): Routing protocol performance issues and evaluation considerations", (Internet-draft). In The internet society, 1999.

[2] C. Perkins, E. Belding-Royer, S. Das, Ad Hoc on-demand Distance Vector (AODV)Routing, Technical Report, 2003.

[3] I.S. AlShawi, L. Yan, W. Pan, B. Luo, Lifetime enhancement in wireless sensor networks using fuzzy approach and a-star algorithm, IEEE Sens. J. 12 (10) (2012) 3010-3018.

[4] G.A. Montoya, Y. Donoso, Energy load balancing strategy to extend lifetime in wireless sensor networks, Procedia Comput. Sci. 17 (2013) 395-402.

[5] R. Kacimi, R. Dhaou, A.-L. Beylot, Load balancing techniques for lifetime maximizing in wireless sensor networks, Ad Hoc Netw. 11 (8) (2013) 2172-2186.

[6] Jha, R., \& Ghosh, D. S. (2018). Energy Efficient Particle Swarm Optimization based Multipath Routing in WSN. INTERNATIONAL JOURNAL ONLINE OF SCIENCE, 4(10), 5. Retrieved from http://ijoscience.com/ojsscience/index.php/ojsscience/article/view/164 . Date accessed: 12 Jan 2019. Doi : https://doi.org/10.24113/ijoscience.v4i10.164 .

[7] Y. Ming Lu, V. WS Wong, An energy-efficient multipath routing protocol for wireless sensor networks, Int. J. Commun. Syst. 20 (7) (2007) 747-766.

[8] T. Liu, Q. Li, P. Liang, An energy-balancing clustering approach for gradient-based routing in wireless sensor networks, Comput Commun 35 (17) (2012) 2150-2161.

[9] Abdelkader Laouida, Abdelnasser Dahmani, Ahcène Bounceur, Reinhardt Euler, Farid Lalem, AbdelkamelTari, "A distributed multi-path routing algorithm to balance energy consumption in wireless sensor networks”, Elsevier, 2017.

Citation: Santosh Verma, (2019 Analysis of MPSO-Successive Interference Cancellation Multipath Routing Protocol. International Journal of Research Studies in Computer Science and Engineering (IJRSCSE), 6(3), pp.1-6. http://dx.doi.org/10.20431/2349-4859.0603001

Copyright: (C) 2019 Authors, this is an open-access article distributed under the terms of the Creative Commons Attribution License, which permits unrestricted use, distribution, and reproduction in any medium, provided the original author and source are credited. 